\title{
Treatment of patients with "high grade" extremity localized chondrosarcoma. Preliminary results
}

\author{
Milan Samardziski ${ }^{1}$, George Zafiroski ${ }^{1}$, Cveta Tolevska ${ }^{2}$, \\ Maja Kalicanin-Markovska ${ }^{1}$, Danica Doncovska ${ }^{1}$, Vesna Anevska ${ }^{1}$, \\ Mirjana Runceva ${ }^{1}$
}

${ }^{1}$ Department for Musculoskeletal Tumours, Clinic for Orthopaedic Surgery, Skopje

${ }^{2}$ Institute of Radiotherapy and Oncology, Skopje, Republic of Macedonia

\begin{abstract}
Background. The treatment of high-grade chondrosarcoma consists of either amputation or radical surgical resection of the tumour. Introducing protocols with neo-adjuvant chemotherapy improves survival rates in patients with a high-grade chondrosarcoma.

Patients and methods. In 7 years period (2000-2006), 18 patients with chondrosarcoma were treated. Female were twice more than male (12/6). Patients age varied from 14 to 73 years (mean 51). Thirteen patients (72\%) had extremities localised chondrosarcoma. Eight patients (8/13), or 62\% with extremity localised high-grade chondrosarcoma were subjected to Scandinavian Sarcoma Group XVI chemotherapy protocol. Six of these patients (75\%) complied with the criteria for limb salvage.
\end{abstract}

Results. The follow up was from 13 months to 8 years (mean 32 months). Two patients (2/8) or 25\% had primarily amputated limb. After the hystopathological assessment of the resected high-grade chondrosarcoma, 6/8 patients (75\%) had bad response to neoadjuvant chemotherapy. There was local recurrence in $3 / 8$ (37\%) and metastases developed in 6/8 (75\%) of the operated patients with high-grade chondrosarcoma. Two of 6 patients (33\%) with limb-salvage had amputations due to local recurrence. Two patients (2/8), or $25 \%$, survived more than 5 years, and up to date they are disease-free.

Conclusions. Patients with extremities localised high-grade chondrosarcoma had higher survival rates and better functional outcome, compared to those with chondrosarcoma localised centrally.

Key words: chondrosarcoma; limb-sparing; neo-adjuvant chemotherapy

\section{Introduction}

Received 23 January 2009

Accepted 8 April 2009

Correspondence to: Prim. Dr. Milan Samardziski MSc, Clinic for Orthopedic Surgery, Vodnjanska 17, 1000

Skopje, Republic of Macedonia. Phone: +38923147626;

E-mail: milan_samardziski@yahoo.com
Chondrosarcomas are very rare tumours. ${ }^{1}$ Their incidence varies from 2 to 3 cases on 1000000 inhabitants. ${ }^{2,3}$ They usually appear in the $2^{\text {nd }}$ or in the $4^{\text {th }}$ decade of life, affecting active and most productive patients. ${ }^{4,5}$ It is well known that they poorly react to chemotherapy, therefore, the radical surgical resection is essential in their 
treatment. There are very few published data on the survival, efficacy and role of chemotherapy of high-grade chondrosarcoma patients. ${ }^{6,7}$ This facts were the motives for this study, and the aim was to analyse the chemotherapy protocol and various limb-salvage surgical techniques in correlation to survival of patients and function of spared limbs.

\section{Patients and methods}

In 7 years period (2000-2006), on the Clinic for Orthopaedic Surgery in Skopje, 18 patients with chondrosarcoma were treated. Female patients were twice more than male (12:6). Patients age varied from 14 to 73 years (mean 51).

Five of these patients, or $28 \%$, had a central localised chondrosarcoma ( 3 on the pelvic ring, 1 on the rib and 1 on the scapula). The rest 13 patients had chondrosarcoma localised on extremities (Figure 1). Eight patients $(8 / 13)$, or $44 \%$ with extremity localised high-grade chondrosarcoma were subjected to Scandinavian Sarcoma Group XVI chemotherapy protocol. Six of this patients $(75 \%)$ complied with the criteria for limb salvage (Enneking stage IIA and IIB). ${ }^{8}$

Only 8 of 18 patients (44\%), with highgrade chondrosarcoma were included in the further analysis. These patients must comply with following inclusion or exclusion criteria.

\section{Inclusion criteria}

The selection of patients for the study inclusion was based on the following criteria:

Histopathologically proven high-grade osteosarcoma (grade III or IV); with no evidence of lung or other metastases; patient age between 8 and 65 years; normal hepatic and renal function; leukocyte count over $3.0 \times 10^{9} / \mathrm{L}$ and platelet count over

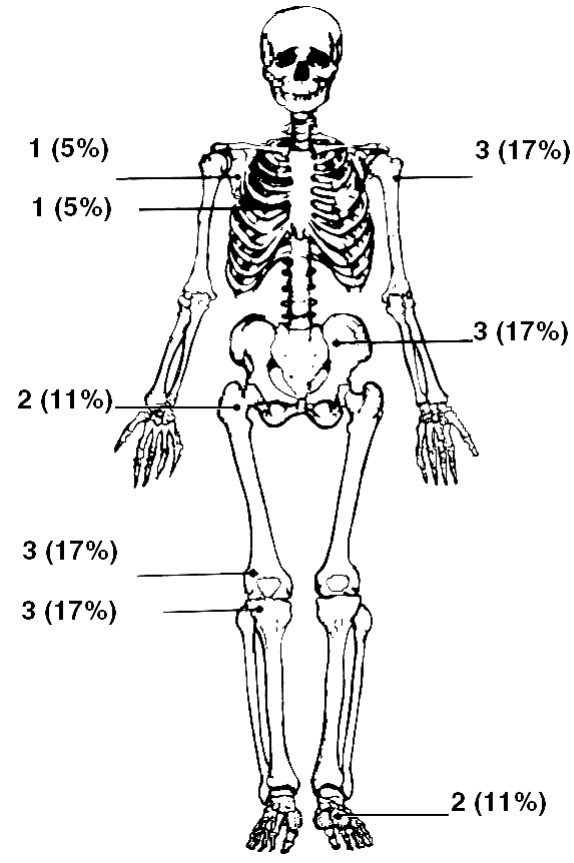

Figure 1. Anatomic distribution of chondrosarcoma in the patients.

$100 \times 10^{9} / \mathrm{L}$; neo-adjuvant chemotherapy was introduced not longer than 1 month after the histological diagnosis of osteosarcoma.

\section{Exclusion criteria}

Exclusion criteria were: evidence of lymphatic or haematogenous metastases at the time of diagnosis; patients under 8 years or older than 65 years; pregnant or a nursing women.

\section{Diagnostic procedures and treatment}

The diagnosis was made by clinical examination, plain X-rays, CT, MRI and histopathologically with open biopsy. Staging was done with Tc $99 \mathrm{~m}$ bone scan, chest X-rays and CT, and CT of the region with osteosarcoma. For the preoperative planning, MRI and standard or CT arteriography were carried out. After the completion 


\section{Scandinavian osteosarcoma protocol, SSG XIV}

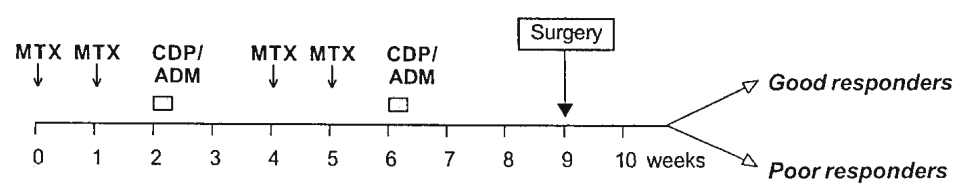

Good responders

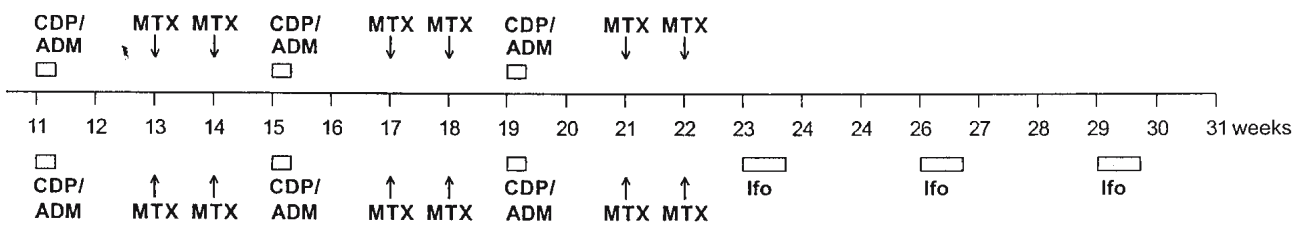

Poor responders

Figure 2. Scandinavian Sarcoma Group protocol (SSG XIV).

of the chemotherapy protocol, clinical and radiographic evaluation of the patients was done every 3 months in the first 3 years and twice a year thereafter.

Eight of 18 patients (with high-grade chondrosarcoma) were administered to the Scandinavian Sarcoma Group XIV neoadjuvant chemotherapy protocol (SSG XIV). Patients received 2 cycles of preoperative chemotherapy (high dose methotrexate $1200 \mathrm{mg} / \mathrm{m}^{2}$, cisplatin $45 \mathrm{mg} / \mathrm{m}^{2} /$ x 2 days and doxorubicin $75 \mathrm{mg} / \mathrm{m}^{2}$; (Figure 2). The surgical resection of the osteosarcoma was made 9 weeks after the beginning of neoadjuvant chemotherapy.

After the resection, a detailed histopathological assessment of the specimen was done to determine the extent of necrosis of the tumour tissue. Considering the percentage of necrotic tumour tissue, patients were classified into two groups. The first group was with good response to chemotherapy ( $>90 \%$ necrosis of the tumour). The second

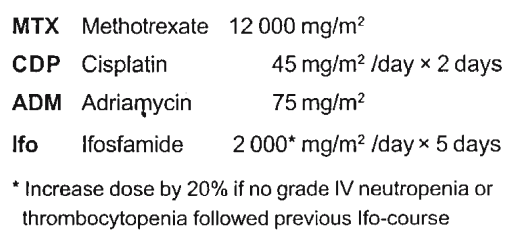

group was with a poor response to chemotherapy ( $>10 \%$ viable tumour). Regarding good or poor response of the tumour to chemotherapy, patients followed different branches of the protocol. All 8/18 patients received 3 courses of postoperative chemotherapy (the same as preoperative). After the hystopathological assessment of resected high-grade chondrosarcoma, $6 / 8$ patients $(75 \%)$ had bad response to neoadjuvant chemotherapy. Patients with a poor response received 3 more cycles of chemotherapy with a high dose Ifosfamide $2000 \mathrm{mg} / \mathrm{m}^{2} /$ day x 5 days plus uromitexan (Ifosfamide: uromitexan $=1: 2$ ). The course was repeated every 3 weeks (Figure 2). 7,9

For the patients who could not satisfy the principles of limb preservation, the ablative surgery was taken into consideration. For those patients the disarticulation of the hip or shoulder griddle, femoral or below the knee, humeral or other amputations were more appropriate..$^{2-4,8,9}$ When tumour- 

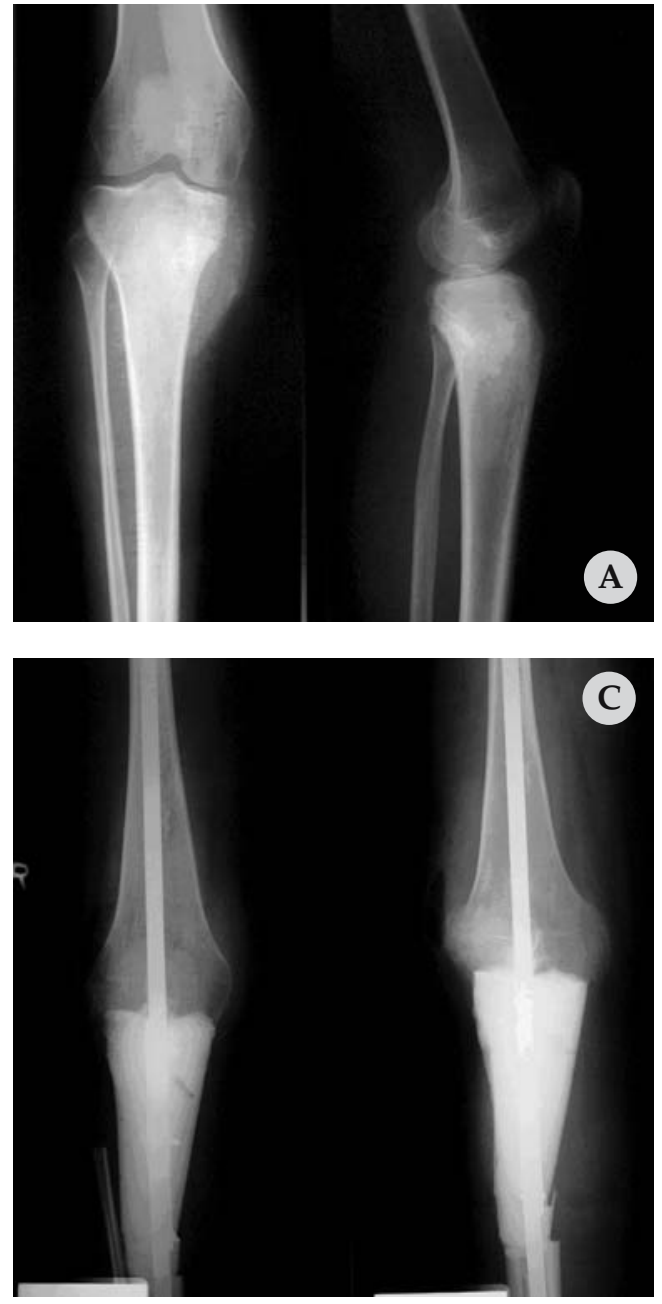

free margins were obtained, a large skeletal defect was often present, requiring the reconstruction of a bone, muscles, other soft tissues, and skin. Patients' age, tumour location and extent of resection narrowed the list of appropriate surgical alternatives. ${ }^{10,11}$

Several options for limb-sparing procedures were available: ${ }^{12}$

- resection arthrodesis and other special indication techniques (Figures 3a-c), 3,11

- modular or special expanding endoprosthesis (Figures 3d, 3f), 13,14

- cortico-spongious or bulk allograft (Figure 3e). ${ }^{13,15}$

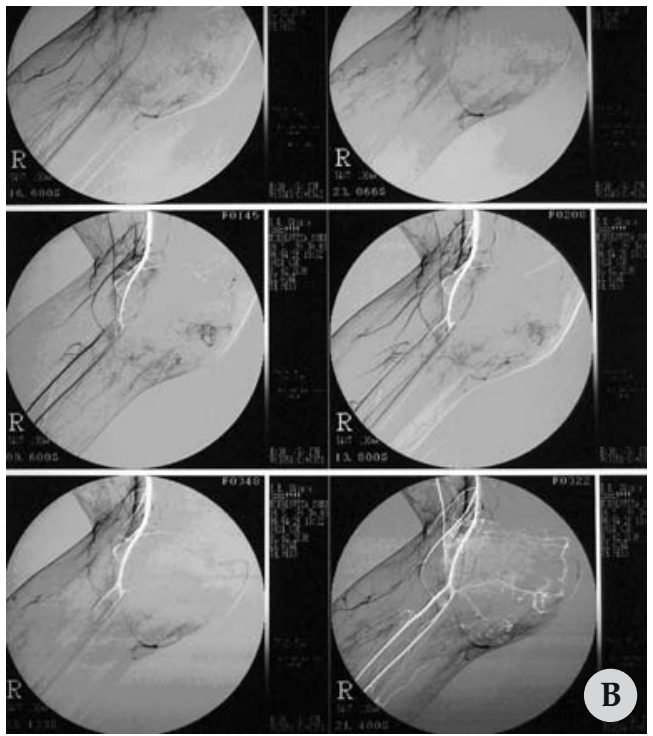

Figures 3 A,B,C. Preoperative X-ray, arteriography and postoperative $\mathrm{X}$-ray of a patient with high-grade chondrosarcoma of proximal tibia, after resection reconstructed with modified Campanacci method.

Musculoskeletal Tumour Society score (MSTSS), based on the Enneking' system for the functional evaluation of reconstructive procedures, was used to determine the functional results. This scoring system evaluates pain, function, patient's emotional acceptance (pertinent to a patient as a whole) and specific factors for evaluating the upper limb (range of motion, manual dexterity and lifting ability) or the lower limb (need of support with orthopaedic accessories, ability to walk and gait). For each of 6 factors, values from 0 to 5 are assigned, with total of 30 (or $100 \%$ function of the limb). For each factor, values 1, 3 and 5 are equated with criteria levels of the achievement or performance. Intermediate values of 2 or 4 are assigned, based on the examiner's judgment, when the achievement or performance falls between the specified values. It is recommended results to be reported numerically in percentage of the normal function (last column in Table 1). ${ }^{16}$

Results were updated in December 2008. 

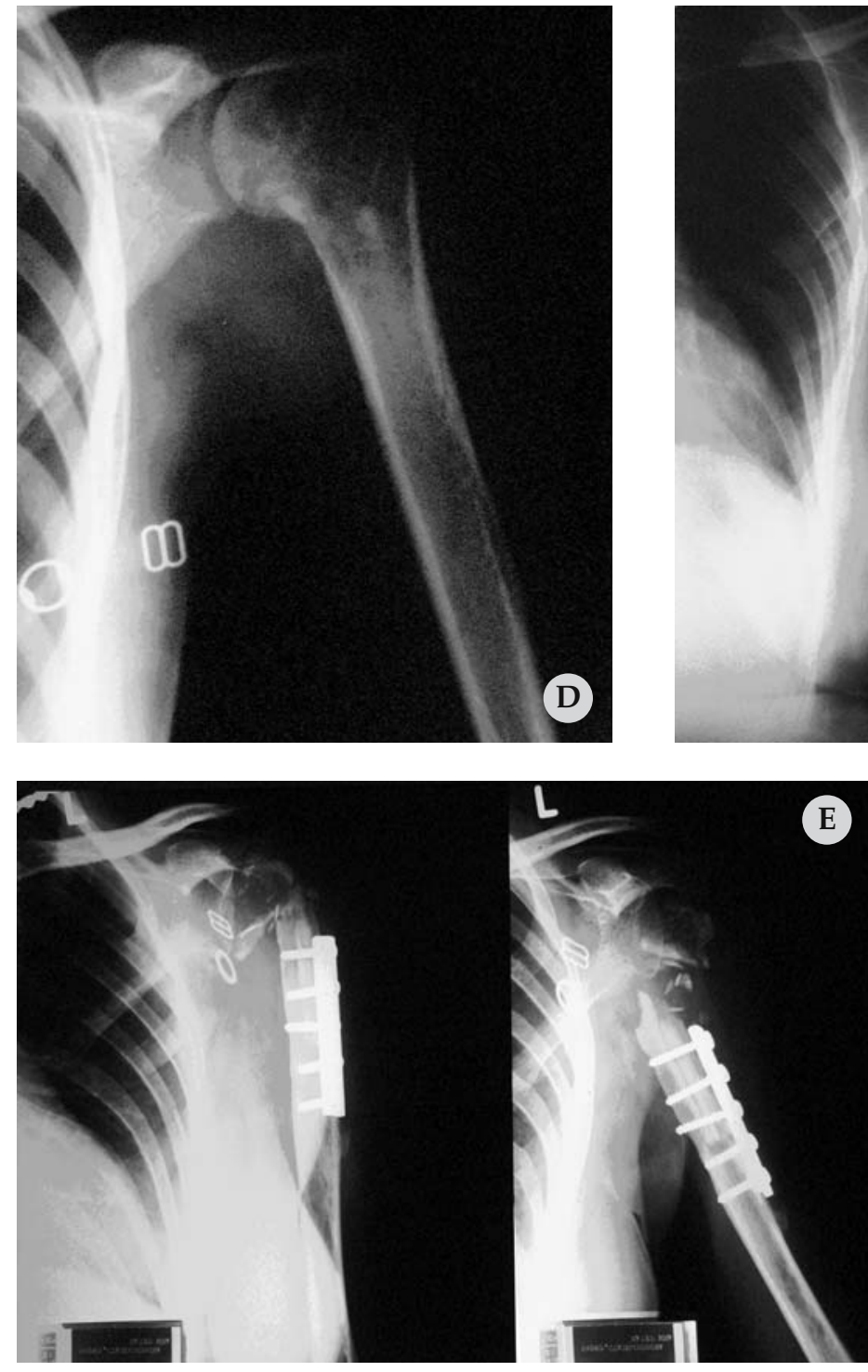

\section{Results}

The follow-up was 2-8 years (mean $36 \pm$ 13 months). Eight of 18 patients (44\%) were with a high-grade chondrosarcoma. Clinical data of patients with high-grade chondrosarcoma treated with chemotherapy and surgery are shown in Table 1 . Two $(2 / 8)$ patients $(25 \%)$ had primarily amputated limb. Six (6/8) patients or $75 \%$ comply

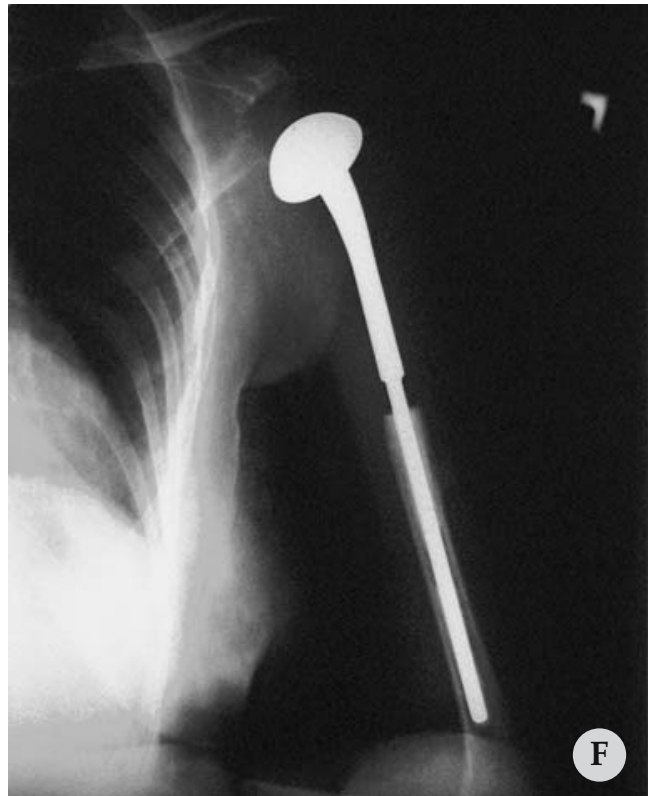

Figures 3 D,E,F. X-ray of a patient with high-grade chondrosarcoma of proximal humerus; fracture of the reconstruction with fibular graft 16 months after the operation and x-ray after implantation of special tumour endoprsthesis in the same patient. with criteria for limb-salvage. After the hystopathological assessment of a resected high-grade chondrosarcoma, 6/8 patients $(75 \%)$ had a bad response to neoadjuvant chemotherapy. Six (6/8) patients, or $75 \%$ had minor or major postoperative complications. Complications developed in period of 1 to 6 months postoperatively (mean $4 \pm 1$ ). Most of the complications were seen after the $4^{\text {th }}$ month. 
Table 1. Clinical data of patients with a high-grade chondrosarcoma treated with chemo and surgery.

\begin{tabular}{|c|c|c|c|c|c|c|c|c|c|}
\hline & Age & $\begin{array}{l}\text { Gender } \\
(\mathrm{M} / \mathrm{F})\end{array}$ & $\begin{array}{l}\text { Compli- } \\
\text { cations }\end{array}$ & $\begin{array}{l}\text { Recurrence } \\
\text { (months) }\end{array}$ & $\begin{array}{l}\text { Metastases } \\
\text { (months) }\end{array}$ & Reoperat. & $\begin{array}{l}\text { Died after } \\
\text { (months) }\end{array}$ & $\begin{array}{l}\text { Follow-up } \\
\text { (months) }\end{array}$ & $\begin{array}{l}\text { MSTSS } \\
(\%)^{*}\end{array}$ \\
\hline \multicolumn{10}{|c|}{ Patients with limb-salvage } \\
\hline 1 & 51 & $\mathrm{~F}$ & 2 & 17 & 35 & Yes & 44 & 44 & $47 \%$ \\
\hline 2 & 14 & $\mathrm{~F}$ & 0 & 0 & 0 & No & 0 & 67 & $93 \%$ \\
\hline 3 & 56 & $\mathrm{M}$ & 4 & 1 & 4 & Yes & 7 & 7 & $23 \%$ \\
\hline 4 & 65 & $\mathrm{M}$ & 1 & 0 & 0 & Yes & 0 & 99 & $53 \%$ \\
\hline 5 & 45 & $\mathrm{~F}$ & $1+3$ & 10 & 13 & Yes & 47 & 47 & $87 \%$ \\
\hline 6 & 64 & M & 3 & 0 & 16 & Yes & 31 & 31 & $50 \%$ \\
\hline \multicolumn{10}{|c|}{ Patients with amputations } \\
\hline 7 & 65 & $\mathrm{~F}$ & 0 & 0 & 9 & No & 20 & 20 & / \\
\hline 8 & 14 & $\mathrm{~F}$ & 3 & 11 & 15 & No & 21 & 21 & I \\
\hline
\end{tabular}

Complications: 1- loosening or fracture of the reconstruction, 2- vein thrombosis, -skin necrosis 4- lung emboly. *MSTSS-Musculoskeletal Tumour Society Score.

There was local recurrence in $3 / 8$ patients with a high-grade chondrosarcoma, or $37 \%$. The recurrence developed in period of 1 to 19 postoperative months (mean 11 \pm 7$)$. Most of the recurrences developed after the $10^{\text {th }}$ month (Figure 4 ). Two of 6 patients (33\%) with limb-salvage had amputations due to local recurrence.

Metastases developed in 6/8 of the patients $(75 \%)$ with high-grade chondrosarco$\mathrm{ma}$, in period of 1 to 35 months postoperatively (mean $17 \pm 10$ ). Most of the metastases developed after the $16^{\text {th }}$ month (Figure 4). Patients who developed metastases died in the period of 13 months to 4 years postoperatively (mean $28 \pm 14$ moths).

Two patients $(2 / 8)$, or $25 \%$, survived more than 5 years, and up to date they are disease-free.

Musculoskeletal Tumour Society score was used for the evaluation of the function of the operated patients and results of the operated limbs were shown as percentage of the maximal function. In our study, the

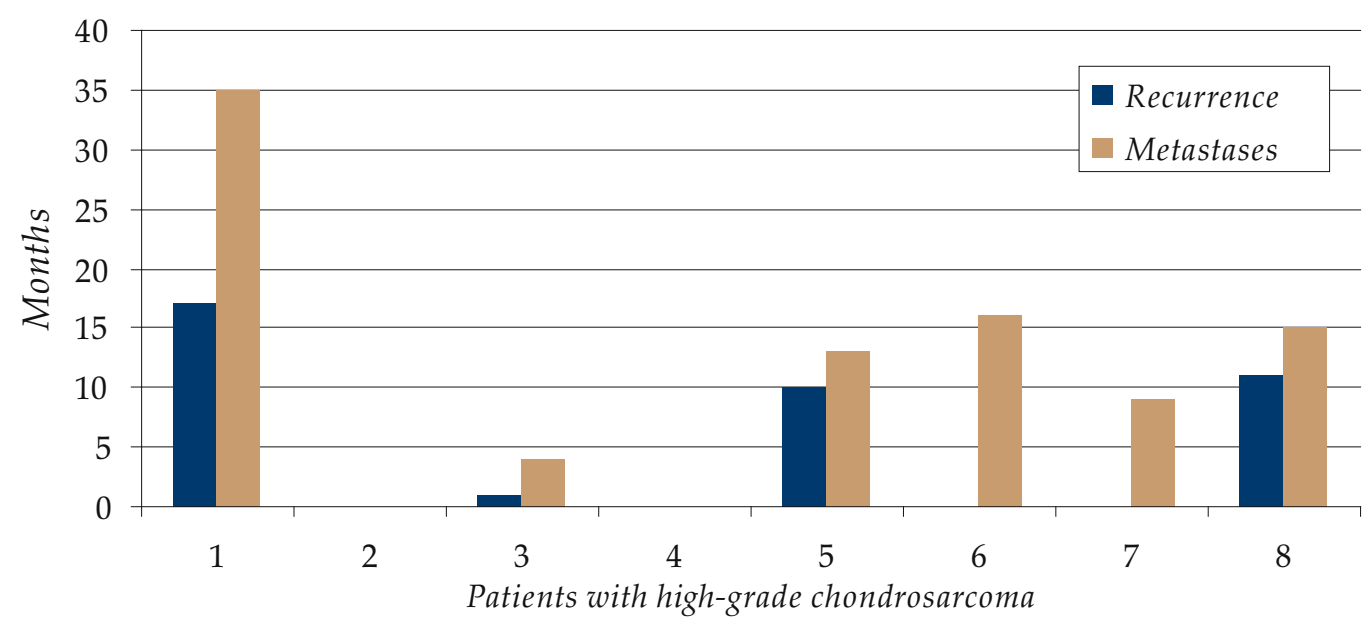

Figure 4. Local recurrence and distant metastases in patients with high-grade chondrosarcoma in our study. 
function after limb-salvage procedures varied from $23 \%$ to $93 \%$ (median $59 \%$ ).

\section{Discussion}

The incidence of local recurrence, metastases and prognosis of the chondrosarcoma treatment are highly variable and hard to predict. $^{3,6}$ Chemo and radiotherapy have low impact on the final outcome. The radical surgical resection of chondrosarcoma is primary treatment. ${ }^{4,5}$ There are very few published data on the survival, efficacy and role of chemotherapy of high-grade chondrosarcoma patients. ${ }^{6,7}$ Lately, some authors separate a high-grade chondrosarcoma as a special diagnostic and treatment problem. ${ }^{10}$ Introducing protocols with a neo-adjuvant chemotherapy should improve survival rates in patients with high-grade chondrosarcoma. $2,7,15$

Our primary goal was to radically extract the tumour, and limb salvage was done only in carefully selected patients. We followed four basic principles of limb-sparing procedures: (1) local recurrence should be no greater and survival no worse than by amputation; (2) the procedure, or treatment of its complications, should not delay the adjuvant therapy; (3) reconstruction should be enduring and not associated with a large number of local complications requiring secondary procedures and frequent hospitalizations; (4) function of the limb should approach that obtained by amputation, although body image, patients preference and life style may influence the decision. $., 10,14,15$ If multidisciplinary approach is accepted, and educated medical stuff in principles of tumour surgery is engaged, more than $50 \%$ 5-year survival is achievable. Similar or better results are reported in many studies. ${ }^{9,10,13-15}$

Eight of 18 patients (44\%) in our study had a high-grade chondrosarcoma and were subjected to Scandinavian Sarcoma Group Protocol XVI. Two (2/8) patients $(25 \%)$ had primarily amputated limb. Six $(6 / 8)$ patients or $75 \%$ comply with criteria for limb-salvage, and 2 of them (33\%) had amputations due to the local recurrence. After the hystopathological assessment of a resected a high-grade chondrosarcoma, 6/8 patients $(75 \%)$ had bad response to neoadjuvant chemotherapy. High percentages of bad response to neoadjuvant chemotherapy are corresponding with results published in some other studies. ${ }^{2,7}$ Two patients (2/8), or $25 \%$, survived more than 5 years, and up to date they are disease-free. Function after limb-salvage procedures in our study varied from $23 \%$ to $93 \%$ (median $59 \%$ ).

\section{Conclusions}

Patients with extremities localised highgrade chondrosarcoma had significantly higher survival rates and better functional outcome, compared to those with chondrosarcoma localised centrally. Introducing protocols with neo-adjuvant chemotherapy improves survival rates in patients with high-grade chondrosarcoma.

\section{References}

1. Kachanov DY, Dobrenkov KV, Shamanskaya TV, Abdullaev RT, Inushkina EV, Savkova RF, et al. Solid tumors in young children in Moscow Region of Russian Federation. Radiol Oncol 2008; 42: 39-44.

2. Lee FY, Mankin JH, Fondren G, Gebhardt MC, Springfild DS, Rosenberg AE, et al. Chondrosarcoma of bone: An assessment of outcome. J Bone Joint Surg Am 1999; 80: 326-38.

3. Zafiroski G. Limb-salvage: dramatic life stories (Macedonian). Skopje: Kultura; 2005. p. 9-12.

4. Brien EW, Mirra JM, Kerr R. Benign and malignant cartilage tumors of bone and joint: their anatomic and theoretical basis with an emphasis on radiology, pathology and clinical biology. Skelet Radiol 1997; 26: 325-53. 
5. Hamilton A, Davis RI, Hayes D, Mollan RAB. Chondrosarcoma developing in synovial chondromatosis. A case report. J Bone Joint Surg 1987; 69: 137-40.

6. Nagarajan R, Neglia PJ, Clohisy DR, Robinson LL. Limb salvage and amputation in survivors of paediatric lower-extremity bone tumours: What are long term implications. J Clin Oncol 2002; 22: 4493-501.

7. Dickey ID, Rose PS, Fuchs B, Wold LE, Okuno SH, Sim FH, et al. Dedifferentiated chondrosarcoma: The role of chemotherapy with updated outcomes. J Bone Joint Surg Am 2004; 86: 2412-8.

8. Enneking WF, Spanier SS, Goodman MA. A surgical staging of musculoskeletal sarcomas. J Bone Joint Surg Am 1980; 62: 1027-30.

9. Bruland OS, Pihl A. On the current management of osteosarcoma. A critical evaluation and proposal for a modified treatment strategy. Eur J Cancer 1997; 33: 1725-31.

10. Di Caprio MR, Friedlander GE. Malignant bone tumors: limb sparing versus amputation. J Amer Acad Ortho Surg 2003; 11: 125-9.
11. Campanacci M. The wrong approach to tumors of muscolo-skeletal system: what should not be done. Chir Organi Mov 1999; 84: 1-17.

12. Čuček-Pleničar M, Novak J, Špiler M, Baebler B, Červek J, Lamovec J. Malignant bone tumours of the extremities: the role of limb sparing surgery. Radiol Oncol 1997; 31: 131-3.

13. Bonardelli S, Nodari F, Maffeis R, Ippolito V, Saccalani M, Lussardi L, et al. Limb salvage in lover-extremity sarcomas and technical details about vascular reconstruction. J Orthop Sci 2000; 5: 555-60.

14. Kotz R, Ritchl P, Trachtenbrodt J. A modular femur-tibia reconstruction system. Orthopaedics 1986; 9: 1639-52.

15. Mochizuki K, Yamagouchi H, Umeda T. The management of pelvic chondrosarcoma. Int Orthop 2000; 24: 65-70.

16. Enneking WF, Dunham W, Gephardt MC, Malawar M, Prichard DJ. A system for functional evalluation of reconstructive procedures after surgical treatment of tumors of the musculoskeletal system. Clin Orthop 1993; 286: 241-6. 